\title{
BOUNDS ON REAL EIGENVALUES AND SINGULAR VALUES OF INTERVAL MATRICES*
}

\author{
MILAN HLADÍK ${ }^{\dagger}$, DAVID DANEY ${ }^{\ddagger}$, AND ELIAS TSIGARIDAS T $^{\ddagger}$
}

\begin{abstract}
We study bounds on real eigenvalues of interval matrices, and our aim is to develop fast computable formulae that produce as-sharp-as-possible bounds. We consider two cases: general and symmetric interval matrices. We focus on the latter case, since on the one hand such interval matrices have many applications in mechanics and engineering, and on the other hand many results from classical matrix analysis could be applied to them. We also provide bounds for the singular values of (generally nonsquare) interval matrices. Finally, we illustrate and compare the various approaches by a series of examples.
\end{abstract}

Key words. interval matrix, interval analysis, real eigenvalue, eigenvalue bounds, singular value

AMS subject classifications. $65 \mathrm{G} 40,65 \mathrm{~F} 15,15 \mathrm{~A} 18$

DOI. $10.1137 / 090753991$

1. Introduction. Many real-life problems suffer from diverse uncertainties, for example, due to data measurement errors. Considering intervals instead of fixed real numbers is one possible way to tackle such uncertainties. In this paper, we study real eigenvalues of matrices, the entries of which vary simultaneously and independently inside some given intervals. The set of all possible eigenvalues forms a finite union of several compact real intervals (see Proposition 2.1), and our aim is to compute as-sharp-as-possible bounds for these intervals.

The problem of computing lower and upper bounds for the eigenvalue set is well studied; see, e.g., [3, 10, 17, 27, 28, 29, 30, 32]. In recent years some effort was made in developing and extending diverse inclusion sets for eigenvalues [8,22] such as Gerschgorin discs or Cassini ovals. Even though such inclusion sets are more or less easy to compute and can be extended to interval matrices, the intervals that they produce are big overestimations of the actual ones.

The interval eigenvalue problem has a lot of applications in the field of mechanics and engineering. Let us mention for instance automobile suspension systems [27], mass structures [26], vibrating systems [11], principal component analysis [12], and robotics [5]. In many cases, the properties of a system are given by the eigenvalues (or singular values) of a Jacobian matrix. A modern approach is to consider that the parameters of this matrix vary in a set of continuous states; hence it is useful to consider this matrix as an interval matrix. The propagation of an interval representation of the parameters in the matrix allows us to bound the properties of the system over all its states. This is useful for designing a system, as well as to certify its performance.

Our goal is to revise and improve the existing formulae for bounding eigenvalues of interval matrices. We focus on algorithms that are useful from a practical point

* Received by the editors March 26, 2009; accepted for publication (in revised form) by A. Frommer March 29, 2010; published electronically June 11, 2010.

http://www.siam.org/journals/simax/31-4/75399.html

$\dagger$ Department of Applied Mathematics, Faculty of Mathematics and Physics, Charles University, Malostranské nám. 25, 11800, Prague, Czech Republic (milan.hladik@matfyz.cz), and INRIA SophiaAntipolis Méditerranée, 2004 route des Lucioles, BP 93, 06902 Sophia-Antipolis Cedex, France (Milan.Hladik@sophia.inria.fr).

${ }^{\ddagger}$ INRIA Sophia-Antipolis Méditerranée, 2004 route des Lucioles, BP 93, 06902 Sophia-Antipolis Cedex, France (David.Daney@sophia.inria.fr, Elias.Tsigaridas@sophia.inria.fr).

2116 
of view, meaning that sometimes we sacrifice the accuracy of the results for speed. Nevertheless, the bounds that we derive are sharp enough for almost all practical purposes and are excellent candidates for initial estimates for various iterative algorithms [17].

We assume that the reader is familiar with the basics of interval arithmetic; otherwise we refer the reader to $[2,14,24]$. An interval matrix is defined as

$$
\boldsymbol{A}:=[\underline{A}, \bar{A}]=\left\{A \in \mathbb{R}^{m \times n} ; \underline{A} \leq A \leq \bar{A}\right\},
$$

where $\underline{A}, \bar{A} \in \mathbb{R}^{m \times n}, \underline{A} \leq \bar{A}$, are given matrices. By

$$
A_{c}:=\frac{1}{2}(\underline{A}+\bar{A}), \quad A_{\Delta}:=\frac{1}{2}(\bar{A}-\underline{A}),
$$

we denote the midpoint and the radius of $\boldsymbol{A}$, respectively. A symmetric interval matrix is defined as

$$
\boldsymbol{A}^{S}:=\left\{A \in \boldsymbol{A} \mid A=A^{T}\right\} .
$$

By an inner approximation of a set $\mathcal{S}$ we mean any subset of $\mathcal{S}$, and by an outer approximation of $\mathcal{S}$ we mean a set containing $\mathcal{S}$ as a subset. Our aim is to develop formulae for calculating an outer approximation of the eigenvalue set of a (general or symmetric) interval matrix. Moreover, the following notation will used throughout the paper:

$$
\begin{array}{ll}
|\boldsymbol{v}|=\max \{-\underline{v}, \bar{v}\} & \text { magnitude (absolute value) of an interval } \boldsymbol{v} \\
|\boldsymbol{A}| & \text { magnitude (absolute value) of an interval ma- } \\
& \text { trix } \boldsymbol{A}, \text { i.e., }|\boldsymbol{A}|_{i j}=\left|\boldsymbol{A}_{i j}\right| \\
\operatorname{diag}(v) & \text { a diagonal matrix with entries } v_{1}, \ldots, v_{n} \\
\|A\|_{p}=\max _{x \neq 0} \frac{\|A x\|_{p}}{\|x\|_{p}} & \text { matrix } p \text {-norm } \\
\kappa_{p}(A)=\|A\|_{p}\left\|A^{-1}\right\|_{p} & \text { condition number (in } p \text {-norm) } \\
\sigma_{\max }(A) & \text { maximal singular value of a matrix } A \\
\rho(A) & \text { spectral radius of a matrix } A \\
\lambda^{\operatorname{Re}}(A) & \text { real part of an eigenvalue of a matrix } A \\
\lambda^{\operatorname{Im}}(A) & \text { imaginary part of an eigenvalue of a matrix } A
\end{array}
$$

The paper consists of two parts: the first is devoted to general interval matrices, and the second to symmetric interval matrices. Symmetry causes dependency between interval quantities, but - on the other hand - stronger theorems are applicable. Moreover, bounds of singular values of interval matrices could be obtained as corollaries.

2. General interval matrix. Let $\boldsymbol{A}$ be a square interval matrix, and let

$$
\Lambda:=\{\lambda \in \mathbb{R} ; A x=\lambda x, x \neq 0, A \in \boldsymbol{A}\}
$$

be the set of all real eigenvalues of matrices in $\boldsymbol{A}$.

Proposition 2.1. The set $\Lambda$ is a finite union of compact real intervals.

Proof. Suppose $\Lambda \neq \emptyset$; otherwise we are done. $\Lambda$ is bounded since $|\lambda| \leq$ $\max \left\{\|A\|_{2} ; A \in \boldsymbol{A}\right\}$ for all $\lambda \in \Lambda$. To show the closedness consider a sequence $\lambda_{i} \in \Lambda$, $i=1, \ldots$, converging to $\lambda \in \mathbb{C}$. For every $i$ there is a matrix $A_{i} \in \boldsymbol{A}$ and a vector $x_{i}$ with $\left\|x_{i}\right\|_{2}=1$ such that $A_{i} x_{i}=\lambda_{i} x_{i}$. Choose a subsequence $\left\{i_{\nu}\right\}, \nu=1, \ldots$, such 
that $A_{i_{\nu}}$ converge to $A \in \boldsymbol{A}$ and $x_{i_{\nu}}$ converge to $x$ with Euclidean norm 1. Going to the limit $\nu \rightarrow \infty$ we get $A x=\lambda x$ showing $\lambda \in \Lambda$.

The finiteness follows from Rohn in [29, Theorem 3.4]. It states that every boundary eigenvalue $\lambda \in \partial \Lambda$ is attained for a matrix $A \in \boldsymbol{A}$, which is of the form $A=A_{c}-\operatorname{diag}(y) A_{\Delta} \operatorname{diag}(z)$, where $y, z \in\{ \pm 1\}^{n}$. Therefore there are finitely many boundary eigenvalues in $\Lambda$ and hence also intervals.

Computation of the real eigenvalue set is considered a very difficult task. Even checking whether $0 \in \Lambda$ is an NP-hard problem since it is equivalent to checking regularity of the interval matrix $\boldsymbol{A}$, which is known to be an NP-hard problem [25]. Therefore, we focus on a fast computation of the initial (hopefully sharp enough) outer approximation of $\Lambda$.

For other approaches that estimate $\Lambda$, we refer the reader to $[10,27,32]$.

Some methods do not calculate bounds for the real eigenvalues of $\boldsymbol{A}$; instead they compute bounds for the real parts of the complex eigenvalues. Denote the set of all possible real parts by

$$
\Lambda_{r}:=\left\{\lambda^{\operatorname{Re}} \in \mathbb{R} ; A x=\lambda x, x \neq 0, A \in \boldsymbol{A}\right\} .
$$

As $\Lambda \subseteq \Lambda_{r}$, any outer approximation to $\Lambda_{r}$ works for $\Lambda$ as well.

Let us recall a method proposed in Rohn [30, Theorem 2] that we will improve in what follows.

Theorem 2.2 (see $[30])$. Let

$$
S_{c}:=\frac{1}{2}\left(A_{c}+A_{c}^{T}\right), \quad S_{\Delta}:=\frac{1}{2}\left(A_{\Delta}+A_{\Delta}^{T}\right) .
$$

Then $\Lambda_{r} \subseteq \boldsymbol{\lambda}^{0}:=\left[\underline{\lambda}^{0}, \bar{\lambda}^{0}\right]$, where

$$
\underline{\lambda}^{0}=\lambda_{\min }\left(S_{c}\right)-\rho\left(S_{\Delta}\right), \quad \bar{\lambda}^{0}=\lambda_{\max }\left(S_{c}\right)+\rho\left(S_{\Delta}\right),
$$

and $\lambda_{\min }\left(S_{c}\right), \lambda_{\max }\left(S_{c}\right)$ denote the minimal and maximal eigenvalue of $S_{c}$, respectively.

In most of the cases, the previous theorem provides a good estimation of the eigenvalue set $\Lambda$ (cf. [17]). However, its main disadvantage is the fact that it produces nonempty estimations, even in the case where the eigenvalue set is empty. To overcome this drawback we propose an alternative approach that utilizes the Bauer-Fike theorem $[13,18,33]$.

Theorem 2.3 (see Bauer and Fike, 1960). Let $A, B \in \mathbb{R}^{n \times n}$ and suppose that $A$ is diagonalizable, that is, $V^{-1} A V=\operatorname{diag}\left(\mu_{1}, \ldots, \mu_{n}\right)$ for some $V \in \mathbb{C}^{n \times n}$ and $\mu_{1}, \ldots, \mu_{n} \in \mathbb{C}$. For every (complex) eigenvalue $\lambda$ of $A+B$, there exists an index $i \in\{1, \ldots, n\}$ such that

$$
\left|\lambda-\mu_{i}\right| \leq \kappa_{p}(V) \cdot\|B\|_{p}
$$

For almost all practical cases the 2-norm seems to be the most suitable choice. In what follows we will use the previous theorem with $p=2$.

Proposition 2.4. Let $A_{c}$ be diagonalizable, i.e., $V^{-1} A_{c} V$ is diagonal for some $V \in \mathbb{C}^{n \times n}$. Then $\Lambda_{r} \subseteq\left(\bigcup_{i=1}^{n} \boldsymbol{\lambda}_{i}\right)$, where for each $i=1, \ldots, n$,

$$
\begin{aligned}
& \underline{\lambda}_{i}=\lambda_{i}^{\mathrm{Re}}\left(A_{c}\right)-\sqrt{\left(\kappa_{2}(V) \cdot \sigma_{\max }\left(A_{\Delta}\right)\right)^{2}-\lambda_{i}^{\operatorname{Im}}\left(A_{c}\right)^{2}}, \\
& \bar{\lambda}_{i}=\lambda_{i}^{\mathrm{Re}}\left(A_{c}\right)+\sqrt{\left(\kappa_{2}(V) \cdot \sigma_{\max }\left(A_{\Delta}\right)\right)^{2}-\lambda_{i}^{\operatorname{Im}}\left(A_{c}\right)^{2}},
\end{aligned}
$$

Copyright $@$ ( ) by SIAM. Unauthorized reproduction of this article is prohibited. 
provided that $\left(\kappa_{2}(V) \cdot \sigma_{\max }\left(A_{\Delta}\right)\right)^{2} \geq \lambda_{i}^{\operatorname{Im}}\left(A_{c}\right)^{2}$; otherwise $\boldsymbol{\lambda}_{i}=\emptyset$ for $i=1, \ldots, n$.

Proof. Every $A \in \boldsymbol{A}$ can be written as $A=A_{c}+A^{\prime}$, where $\left|A^{\prime}\right| \leq A_{\Delta}$ (where the inequality applies elementwise). The Bauer-Fike theorem with 2-norm implies that for each complex eigenvalue $\lambda(A)$ there is some complex eigenvalue $\lambda_{i}\left(A_{c}\right)$ such that

$$
\left|\lambda(A)-\lambda_{i}\left(A_{c}\right)\right| \leq \kappa_{2}(V) \cdot\left\|A^{\prime}\right\|_{2}=\kappa_{2}(V) \cdot \sigma_{\max }\left(A^{\prime}\right) .
$$

As $\left|A^{\prime}\right| \leq A_{\Delta}$, we have $\sigma_{\max }\left(A^{\prime}\right) \leq \sigma_{\max }\left(A_{\Delta}\right)$. Hence

$$
\left|\lambda(A)-\lambda_{i}\left(A_{c}\right)\right| \leq \kappa_{2}(V) \cdot \sigma_{\max }\left(A_{\Delta}\right) .
$$

Thus all complex eigenvalues of all matrices $A \in \boldsymbol{A}$ lie in the circles with centers in $\lambda_{i}\left(A_{c}\right)$ 's with corresponding radii $\kappa_{2}(V) \cdot \sigma_{\max }\left(A_{\Delta}\right)$. The formulae $(2.1)-(2.2)$ represent an intersection of these circles with the real axis.

Notice that both a pair of complex conjugate eigenvalues $\lambda_{i}\left(A_{c}\right)$ and $\lambda_{j}\left(A_{c}\right)$ yields the same interval $\boldsymbol{\lambda}_{i}=\boldsymbol{\lambda}_{j}$, so it suffices to consider only one of them.

Proposition 2.4 is a very useful tool for estimating $\Lambda$ in the case where the "large" complex eigenvalues of $A_{c}$ also have large imaginary parts. It is neither provably better nor provably worse than Rohn's theorem; see Example 2.8. Therefore it is advisable, in practice, to use both of them.

Proposition 2.4 can be applied only if $A_{c}$ is diagonalizable. For the case where $A_{c}$ is defective we can build upon a generalization of the Bauer-Fike theorem due to Chu $[6,7]$. We present its special form.

Theorem 2.5 (see [6]). Let $A, B \in \mathbb{R}^{n \times n}$ and let $V^{-1} A V=J$ be the Jordan canonical form of $A$. Denote by $p$ the maximal dimension of the Jordan's blocks in $J$. Then for every (complex) eigenvalue $\lambda$ of $A+B$, there is $i \in\{1, \ldots, n\}$ such that

$$
\left|\lambda-\lambda_{i}(A)\right| \leq \max \left\{\Theta_{2}, \Theta_{2}^{\frac{1}{p}}\right\}
$$

where

$$
\Theta_{2}=\sqrt{\frac{p(p+1)}{2}} \cdot \kappa_{2}(V) \cdot\|B\|_{2} .
$$

Proceeding in a manner similar to that in the proof of Proposition 2.4 we obtain the following general result for interval matrices.

Proposition 2.6. Let $V^{-1} A_{c} V=J$ be the Jordan canonical form of $A_{c}$, and let $p$ be the maximal dimension of the Jordan's blocks in J. Denote

$$
\Theta_{2}=\sqrt{\frac{p(p+1)}{2}} \cdot \kappa_{2}(V) \cdot \sigma_{\max }\left(A_{\Delta}\right), \quad \Theta=\max \left\{\Theta_{2}, \Theta_{2}^{\frac{1}{p}}\right\} .
$$

Then $\Lambda \subseteq\left(\bigcup_{i=1}^{n} \boldsymbol{\lambda}_{i}\right)$, where for each $i=1, \ldots, n$,

$$
\begin{aligned}
& \underline{\lambda}_{i}=\lambda_{i}^{\operatorname{Re}}\left(A_{c}\right)-\sqrt{\Theta^{2}-\lambda_{i}^{\operatorname{Im}}\left(A_{c}\right)^{2}}, \\
& \bar{\lambda}_{i}=\lambda_{i}^{\operatorname{Re}}\left(A_{c}\right)+\sqrt{\Theta^{2}-\lambda_{i}^{\operatorname{Im}}\left(A_{c}\right)^{2}},
\end{aligned}
$$

provided that $\Theta^{2} \geq \lambda_{i}^{\operatorname{Im}}\left(A_{c}\right)^{2}$; otherwise $\boldsymbol{\lambda}_{i}=\emptyset$.

This result is applicable for any interval matrix $\boldsymbol{A}$. In our experience, Rohn's bounds are usually more narrow when the input intervals of $\boldsymbol{A}$ are wide. On the other hand, this formula is better as long as the input intervals are narrow; cf. Example 2.9. 
We present one more improvement for computing bounds of $\Lambda$, which is based on a theorem by Horn and Johnson [19].

Theorem 2.7. Let $A \in \mathbb{R}^{n \times n}$. Then

$$
\lambda_{\min }\left(\frac{A+A^{T}}{2}\right) \leq \lambda^{\operatorname{Re}}(A) \leq \lambda_{\max }\left(\frac{A+A^{T}}{2}\right)
$$

for every (complex) eigenvalue $\lambda(A)$ of the matrix $A$.

The theorem says that any upper or lower bound of the eigenvalue set of the symmetric interval matrix $\frac{1}{2}\left(\boldsymbol{A}+\boldsymbol{A}^{T}\right)^{S}$ is also a bound of $\Lambda_{r}$. Symmetric interval matrices are studied in detail in section 3 and the results obtained there can be used here to bound $\Lambda$ via Theorem 2.7. Note that in this way, Rohn's bounds from Theorem 3.1 yield the same bounds as those from Theorem 2.2. Note also that if the interval matrix $\boldsymbol{A}$ is pointed (i.e., $\underline{A}=\bar{A}$ ), then Theorems 2.2 and 2.7 yield the same range.

In what follows we present two examples that utilize the bounds of the previous propositions. We should mention that the purpose of all the examples in the present paper is to illustrate the proposed bounds; hence no verified computations were carried out, as should always be the case for real-life applications.

Example 2.8. Let

$$
\boldsymbol{A}=\left(\begin{array}{ccccc}
{[-5,-4]} & {[-9,-8]} & {[14,15]} & {[4.6,5]} & {[-1.2,-1]} \\
{[17,18]} & {[17,18]} & {[1,2]} & {[4,5]} & {[10,11]} \\
{[17,17.2]} & {[-3.5,-2.7]} & {[1.9,2.1]} & {[-13,-12]} & {[6,6.4]} \\
{[18,19]} & {[2,3]} & {[18,19]} & {[5,6]} & {[6,7]} \\
{[13,14]} & {[18,19]} & {[9,10]} & {[-18,-17]} & {[10,11]}
\end{array}\right)
$$

Rohn's theorem provides the outer approximation $\Lambda \subseteq[-22.104,35.4999]$. Now we utilize Proposition 2.4. The eigenvalues of $A_{c}$ are

$$
-15.8973,-4.0671,15.1215+15.9556 i, 15.1215-15.9556 i, \text { and } 20.7214,
$$

while $\kappa_{2}(V) \cdot \sigma_{\max }\left(A_{\Delta}\right)=8.5887$. Hence

$$
\begin{aligned}
& \lambda_{1}=[-24.486,-7.30853], \quad \boldsymbol{\lambda}_{2}=[-12.6559,4.5216], \\
& \boldsymbol{\lambda}_{3}=\boldsymbol{\lambda}_{4}=\emptyset, \quad \boldsymbol{\lambda}_{5}=[12.1327,29.3101] .
\end{aligned}
$$

The resulting outer approximation of $\Lambda$ is a union of two intervals, i.e.,

$$
[-24.486,4.5216] \cup[12.1327,29.3101] .
$$

Proposition 2.6 yields the same result since the eigenvalues of $A_{c}$ are mutually different.

If we take into account the results of all the methods, and we consider the intersection of the corresponding intervals, we obtain a sharper result, i.e., $[-22.104,4.5216] \cup$ [12.1327, 29.3101].

To estimate the quality of the aforementioned results, it is worth noticing that the exact description of the real eigenvalue set of $\boldsymbol{A}$ could be obtained using the algorithm in [17],

$$
\Lambda=[-17.5116,-13.7578] \cup[-6.7033,-1.4582] \cup[16.7804,23.6143] .
$$


Example 2.9. Let $\boldsymbol{A}=\left[A_{c}-A_{\Delta} ; A_{c}+A_{\Delta}\right]$, where

$$
A_{c}=\left(\begin{array}{cccc}
4 & 6 & 13 & 1 \\
-4 & -5 & -16 & -4 \\
1 & 2 & 6 & 1 \\
0 & -2 & -10 & -1
\end{array}\right)
$$

and all entries of $A_{\Delta}$ equal $\varepsilon$. The eigenvalues of $A_{c}$ are $1 \pm 2 i$ (both are double).

Let $\varepsilon=0.01$. Rohn's theorem leads to the outer approximation [-11.9445, 13.8445]. Proposition 2.4 is not applicable as $A_{c}$ is defective. Using Proposition 2.6 we calculate $p=2$ and $\Theta=1.0612$ and conclude that $\Lambda=\emptyset$, i.e., no matrix $A \in \boldsymbol{A}$ has any real eigenvalue.

For $\varepsilon=1$, Rohn's outer approximation is [ $-15.9045,17.8045]$, but Proposition 2.6 results in $[-105.102,107.102]$.

3. Symmetric interval matrix. Let $A \in \mathbb{R}^{n \times n}$ be a real symmetric matrix. It has $n$ real eigenvalues, which are in decreasing order (including multiplicities):

$$
\lambda_{1}(A) \geq \lambda_{2}(A) \geq \cdots \geq \lambda_{n}(A) .
$$

Let $\boldsymbol{A}^{S}$ be a symmetric interval matrix and denote by

$$
\boldsymbol{\lambda}_{i}\left(\boldsymbol{A}^{S}\right):=\left\{\lambda_{i}(A) \mid A \in \boldsymbol{A}^{S}\right\}
$$

the set of the $i$ th eigenvalues. Each of these sets is a compact real interval; this is due to the continuity of the eigenvalue function and the compactness and convexity of $\boldsymbol{A}^{S}$ [16]. It can happen that the sets $\boldsymbol{\lambda}_{i}\left(\boldsymbol{A}^{S}\right)$ and $\boldsymbol{\lambda}_{j}\left(\boldsymbol{A}^{S}\right)$, where $i \neq j$, overlap.

Our aim is to compute as-sharp-as-possible bounds of the eigenvalue sets. The upper bound $\lambda_{i}^{u}\left(\boldsymbol{A}^{S}\right), i \in\{1, \ldots, n\}$, is any real number satisfying $\lambda_{i}^{u}\left(\boldsymbol{A}^{S}\right) \geq \bar{\lambda}_{i}\left(\boldsymbol{A}^{S}\right)$. Lower bounds $\lambda_{i}^{l}\left(\boldsymbol{A}^{S}\right)$ for $\underline{\lambda}_{i}\left(\boldsymbol{A}^{S}\right), i \in\{1, \ldots, n\}$, can be computed as upper bounds of $-\boldsymbol{A}^{S}$, so we omit their treatment.

The symmetric case is very important for real-life applications as symmetric matrices appear very often in engineering problems. Under the concept of interval computations, symmetry induces dependencies between the matrix elements, which are hard to deal with in general. The straightforward approach would be to "forget" the dependencies and apply the methods from the previous section to obtain bounds on eigenvalues. Unfortunately, these bounds are far from sharp, since the loss of dependency implies a big overestimation on the computed intervals.

We should mention that there are very few theoretical results concerning symmetric interval matrices. Let us mention only that computing all the exact boundary points of the eigenvalue set is not known. Such a result could be of extreme practical importance since it can be used for testing the accuracy of existing approximation algorithms. In this line of research, let us mention the work of Deif [10] and Hertz $[15,16]$. The former provides an exact description of the eigenvalue set, but it works only under some not easily verified assumptions on sign pattern invariance of eigenvectors; the latter (see also [31]) proposes a formula for computing the exact extremal values $\underline{\lambda}_{1}\left(\boldsymbol{A}^{S}\right), \bar{\lambda}_{1}\left(\boldsymbol{A}^{S}\right), \underline{\lambda}_{n}\left(\boldsymbol{A}^{S}\right)$, and $\bar{\lambda}_{n}\left(\boldsymbol{A}^{S}\right)$, which consists of $2^{n-1}$ iterations. Theoretical results could also be found in the work of Qiu and Wang [28]. However, some results turned out to be incorrect [34].

Since the exact problem of computing the eigenvalue set(s) is a difficult one, several approximation algorithms were developed in recent years. An evolution strategy method by Yuan, He, and Leng [34] yields an inner approximation of the eigenvalue 
set. By means of matrix perturbation theory, Qiu, Chen, and Elishakoff [26] proposed an algorithm for approximate bounds, and Leng and He [21] for outer approximation. Outer approximation was also presented by Beaumont [4]; he used a polyhedral approximation of eigenpairs and an iterative improvement. Kolev [20] developed an outer approximation algorithm for the general case with nonlinear dependencies.

3.1. Basic bounds. The following theorem (without proof) appeared in [31]; to ensure that this paper is self-contained, we present its proof.

TheOREM 3.1. It holds that

$$
\boldsymbol{\lambda}_{i}\left(\boldsymbol{A}^{S}\right) \subseteq\left[\lambda_{i}\left(A_{c}\right)-\rho\left(A_{\Delta}\right), \lambda_{i}\left(A_{c}\right)+\rho\left(A_{\Delta}\right)\right] .
$$

Proof. By Weyl's theorem [13, 18, 23, 33], for any symmetric matrices $B, C \in$ $\mathbb{R}^{n \times n}$ it holds that

$$
\lambda_{i}(B)+\lambda_{n}(C) \leq \lambda_{i}(B+C) \leq \lambda_{i}(B)+\lambda_{1}(C) \quad \forall i=1, \ldots, n .
$$

Particularly, for every $A \in \boldsymbol{A}$ in the form of $A=A_{c}+A^{\prime}, A^{\prime} \in\left[-A_{\Delta}, A_{\Delta}\right]$, we have

$$
\lambda_{i}(A)=\lambda_{i}\left(A_{c}+A^{\prime}\right) \leq \lambda_{i}\left(A_{c}\right)+\lambda_{1}\left(A^{\prime}\right) \leq \lambda_{i}\left(A_{c}\right)+\rho\left(A^{\prime}\right) \quad \forall i=1, \ldots, n .
$$

As $\left|A^{\prime}\right| \leq A_{\Delta}$, we get $\rho\left(A^{\prime}\right) \leq \rho\left(A_{\Delta}\right)$, whence

$$
\lambda_{i}(A) \leq \lambda_{i}\left(A_{c}\right)+\rho\left(A_{\Delta}\right) .
$$

Working similarly, we can prove that $\lambda_{i}(A) \geq \lambda_{i}\left(A_{c}\right)-\rho\left(A_{\Delta}\right)$.

The bounds obtained by the previous theorem are usually quite sharp. However, the main drawback is that all the produced intervals $\boldsymbol{\lambda}_{i}\left(\boldsymbol{A}^{\dot{S}}\right), 1 \leq i \leq n$, have the same width.

The following proposition provides an upper bound for the largest eigenvalue of $\boldsymbol{A}^{S}$, i.e., an upper bound for the right endpoint of $\boldsymbol{\lambda}_{1}\left(\boldsymbol{A}^{S}\right)$. Even though the formula is very simple and the bound is not very sharp, there are cases where it yields a better bound than the one obtained by Rohn's theorem. In particular it provides better bounds for nonnegative interval matrices and for interval matrices such as the ones we consider in subsection 3.3 with the form $\left[-A_{\Delta}, A_{\Delta}\right]$.

Proposition 3.2. It holds that

$$
\bar{\lambda}_{1}\left(\boldsymbol{A}^{S}\right) \leq \lambda_{1}(|\boldsymbol{A}|)
$$

Proof. Using the well-known Courant-Fischer theorem [13, 18, 23, 33], we have for every $A \in \boldsymbol{A}$

$$
\begin{aligned}
\lambda_{1}(A) & =\max _{x^{T} x=1} x^{T} A x \leq \max _{x^{T} x=1}\left|x^{T} A x\right| \\
& \leq \max _{x^{T} x=1}|x|^{T}|A||x| \leq \max _{x^{T} x=1}|x|^{T}|\boldsymbol{A}||x| \\
& =\max _{x^{T} x=1} x^{T}|\boldsymbol{A}| x=\lambda_{1}(|\boldsymbol{A}|) .
\end{aligned}
$$

In the same way we can compute a lower bound for the eigenvalue set of $\boldsymbol{A}$ : $\underline{\lambda}_{n}\left(\boldsymbol{A}^{S}\right) \geq-\lambda_{1}(|\boldsymbol{A}|)$. However, this inequality is not so useful in practice. 
3.2. Interlacing approach, direct version. The approach that we propose in this section is based on Cauchy's interlacing property for eigenvalues of a symmetric matrix $[13,18,23,33]$.

Theorem 3.3 (interlacing property; see Cauchy, 1829). Let $A \in \mathbb{R}^{n}$ be a symmetric matrix, and let $A_{i}$ be a matrix obtained from $A$ by removing the ith row and column. Then

$$
\lambda_{1}(A) \geq \lambda_{1}\left(A_{i}\right) \geq \lambda_{2}(A) \geq \lambda_{2}\left(A_{i}\right) \geq \cdots \geq \lambda_{n-1}\left(A_{i}\right) \geq \lambda_{n}(A) .
$$

We develop two methods based on the interlacing property, the direct and the indirect one. These methods are useful as long as the intervals $\boldsymbol{\lambda}_{i}\left(\boldsymbol{A}^{S}\right), i=1, \ldots, n$, do overlap, or as long as there is a narrow gap between them. Overlapping happens, for example, when there are multiple eigenvalues in $\boldsymbol{A}^{S}$. If none of the previous cases occur, then the bounds are not so sharp; see Example 3.6.

The first method uses the interlacing property directly. Bounds on the eigenvalues of the principal minor $\boldsymbol{A}_{i}^{S}$ are also bounds on the eigenvalues of matrices in $\boldsymbol{A}^{S}$ (except for $\bar{\lambda}_{1}\left(\boldsymbol{A}^{S}\right)$ and $\underline{\lambda}_{n}\left(\boldsymbol{A}^{S}\right)$ ). The basic idea is to compute the bounds recursively. However, such a recursive algorithm would be of exponential complexity. Therefore, we propose a simple local search approach that requires only a linear number of iterations and the results of which are quite satisfactory. It consists of selecting the most promising principal minor $\boldsymbol{A}_{i}$ and recursively using only this. To obtain even better results in practice, we apply this procedure in the reverse order as well. (That is, we begin with some diagonal element $\boldsymbol{a}_{i i}$ of $\boldsymbol{A}^{S}$, which is a matrix one-by-one, and iteratively increase its dimension until we obtain $\boldsymbol{A}^{S}$.)

The algorithmic scheme is presented in Algorithm 1. We often need to compute an upper bound $\lambda_{1}^{u}\left(\boldsymbol{B}^{S}\right)$ for the maximal eigenvalue of any matrix in $\boldsymbol{B}^{S}$ (steps 3 and 12). For this purpose we can call Theorem 3.1 or Proposition 3.2, or, to obtain the best results, we choose the minimum of the two. Notice that the algorithm computes only upper bounds for $\bar{\lambda}_{i}\left(\boldsymbol{A}^{S}\right), i=1, \ldots, n$. Lower bounds for $\underline{\lambda}_{i}\left(\boldsymbol{A}^{S}\right), i=1, \ldots, n$, can be obtained by calling the algorithm using $-\boldsymbol{A}^{S}$ as input matrix.

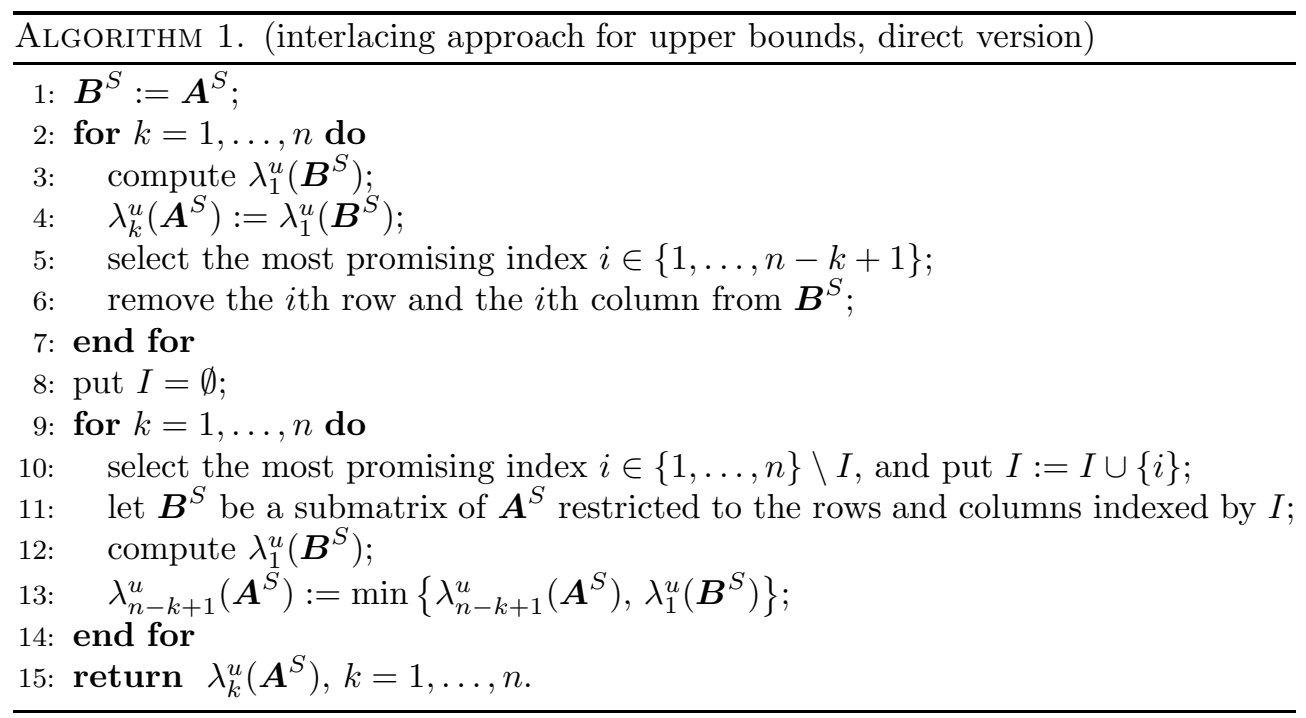

An important ingredient of the algorithm is the selection of index $i$ in steps 5 and 10 . We describe the selection for step 5 ; for step 10 we work similarly. In essence, 
there are two basic choices:

$$
i:=\arg \min _{j=1, \ldots, n-k+1} \lambda_{1}^{u}\left(\boldsymbol{B}_{j}^{S}\right),
$$

and

$$
i:=\arg \min _{j=1, \ldots, n-k+1} \sum_{r, s \neq j}\left|\boldsymbol{B}_{r, s}\right|^{2} .
$$

In both cases we select an index $i$ in order to minimize $\bar{\lambda}_{1}\left(\boldsymbol{B}_{i}^{S}\right)$.

The first formula requires more computation than the second but yields the optimal index in more cases than the second. The latter formula is based on the wellknown result $[18,33]$ that the square of the Frobenius norm of a normal matrix (i.e., the sum of squares of its entries) equals the sum of squares of its eigenvalues. Therefore, the most promising index is the one that maximizes the sum of squares of the absolute values (magnitudes) of the removed components.

The selection rule (3.1) causes a quadratic time complexity of Algorithm 1 with respect to the number of calculations of spectral radii or eigenvalues. Using the selection rule (3.2) results in only a linear number of such calculations.

3.3. Interlacing approach, indirect version. The second method also uses the interlacing property and is based on the following idea. Every matrix $A \in \boldsymbol{A}^{S}$ can be written as $A=A_{c}+A_{\delta}$ with $A_{\delta} \in\left[-A_{\Delta}, A_{\Delta}\right]^{S}$. We compute the eigenvalues of the real matrix $A_{c}$ and bounds on eigenvalues of matrices in $\left[-A_{\Delta}, A_{\Delta}\right]^{S}$, and we "merge" them to obtain bounds on eigenvalues of matrices in $\boldsymbol{A}^{S}$. For the "merging" step we use a theorem for perturbed eigenvalues.

The algorithm is presented in Algorithm 2. It returns only upper bounds $\lambda_{i}^{u}\left(\boldsymbol{A}^{S}\right)$, $i=1, \ldots, n$ for $\bar{\lambda}_{i}\left(\boldsymbol{A}^{S}\right), i=1, \ldots, n$, since lower bounds are likewise computable. The bounds required in step 2 are computed using Algorithm 1.

The following theorem due to Weyl $[18,33]$ gives very nice formulae for the eigenvalues of a matrix sum, which we use in step 4 of Algorithm 2.

Theorem 3.4 (Weyl, 1912). Let $A, B \in \mathbb{R}^{n \times n}$ be symmetric matrices. Then

$$
\begin{array}{ll}
\lambda_{r+s-1}(A+B) \leq \lambda_{r}(A)+\lambda_{s}(B) & \forall r, s \in\{1, \ldots, n\}, r+s \leq n+1, \\
\lambda_{r+s-n}(A+B) \geq \lambda_{r}(A)+\lambda_{s}(B) & \forall r, s \in\{1, \ldots, n\}, r+s \geq n+1 .
\end{array}
$$

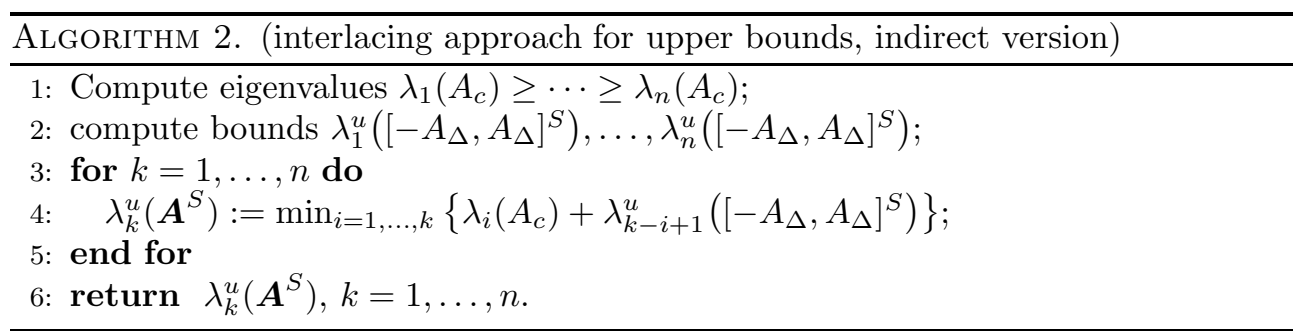

3.4. Diagonal maximization. In this subsection we show that the largest eigenvalues are achieved when the diagonal entries of $A \in A^{S}$ are the maximum entries. Therefore, we can fix them and consider only a subset of $A \in \boldsymbol{A}^{S}$. Similar results can be obtained for the smallest eigenvalues. 
Lemma 3.5. Let $i \in\{1, \ldots, n\}$. Then there is some matrix $A \in A^{S}$ with diagonal entries $A_{j, j}=\bar{A}_{j, j}$ such that $\lambda_{i}(A)=\bar{\lambda}_{i}\left(\boldsymbol{A}^{S}\right)$.

Proof. Let $A^{\prime} \in \boldsymbol{A}^{S}$ be such that $\lambda_{i}\left(A^{\prime}\right)=\bar{\lambda}_{i}\left(\boldsymbol{A}^{S}\right)$. Such a matrix always exists, since $\bar{\lambda}_{i}\left(\boldsymbol{A}^{S}\right)$ is defined as the maximum of a continuous function on a compact set. We define $A \in \boldsymbol{A}^{S}$ as follows: $A_{i j}:=A_{i j}^{\prime}$ if $i \neq j$, and $A_{i j}:=\bar{A}_{i j}$ if $i=j$. By the Courant-Fischer theorem [13, 18, 23, 33], we have

$$
\begin{aligned}
\lambda_{i}\left(A^{\prime}\right) & =\max _{V \subseteq \mathbb{R}^{n} ; \operatorname{dim} V=i} \min _{x \in V ; x^{T} x=1} x^{T} A^{\prime} x \\
& \leq \max _{V \subseteq \mathbb{R}^{n} ; \operatorname{dim} V=i} \min _{x \in V ; x^{T} x=1} x^{T} A x \\
& =\lambda_{i}(A) .
\end{aligned}
$$

Hence $\lambda_{i}(A)=\lambda_{i}(A)^{\prime}=\bar{\lambda}_{i}\left(\boldsymbol{A}^{S}\right)$.

This lemma implies that for computing upper bounds $\lambda_{i}^{u}\left(\boldsymbol{A}^{S}\right)$ of $\bar{\lambda}_{i}\left(\boldsymbol{A}^{S}\right), i=$ $1, \ldots, n$, it suffices to consider only the symmetric interval matrix $\boldsymbol{A}_{r}^{S} \subseteq \boldsymbol{A}^{S}$ defined as

$$
\boldsymbol{A}_{r}^{S}:=\left\{A \in \boldsymbol{A}^{S} \mid A_{j, j}=\bar{A}_{j, j} \forall j=1, \ldots, n\right\} .
$$

To this interval matrix we can apply all the algorithms developed in the previous subsections. The resulting bounds are sometimes sharper and sometimes not so sharp; see Examples 3.6-3.7. So the best possible results are obtained by using all the methods together.

3.5. Singular values. Let $A \in \mathbb{R}^{m \times n}$ and denote $q:=\min \{m, n\}$. By $\sigma_{1}(A) \geq$ $\cdots \geq \sigma_{n}(A)$ we denote the singular values of $A$. It is well known [13, 18, 23] that the singular values of $A$ are identical with the $q$ largest eigenvalues of the Jordan-Wielandt matrix

$$
\left(\begin{array}{cc}
0 & A^{T} \\
A & 0
\end{array}\right)
$$

which is symmetric. Consider an interval matrix $\boldsymbol{A} \subset \mathbb{R}^{m \times n}$. By $\boldsymbol{\sigma}_{i}(\boldsymbol{A}):=\left\{\sigma_{i}(A) \mid\right.$ $A \in \boldsymbol{A}\}, i=1, \ldots, q$, we denote the singular value sets of $\boldsymbol{A}$. The problem of approximating the singular value sets was considered, e.g., in [1,9]. Deif's method [9] produces exact singular value sets, but only under some assumptions that are generally difficult to verify. Ahn and Chen [1] presented a method for calculating the largest possible singular value $\bar{\sigma}_{1}(\boldsymbol{A})$. It is a slight modification of [15] and its time complexity is exponential ( $2^{m+n-1}$ iterations). They also proposed a lower bound calculation for the smallest possible singular value $\underline{\sigma}_{n}(\boldsymbol{A})$ by means of interval matrix inversion.

To get an outer approximation of the singular value set of $\boldsymbol{A}$ we can exhibit the methods proposed in the previous subsections and apply them on the eigenvalue set of the symmetric interval matrix

$$
\left(\begin{array}{cc}
0 & \boldsymbol{A}^{T} \\
\boldsymbol{A} & 0
\end{array}\right)^{S}
$$

Diagonal maximization (see subsection 3.4) has no effect, since the diagonal of the symmetric interval matrix (3.3) consists of zeros only. The other methods work well. Even though they run very fast, they can be accelerated a bit, as some of them can be slightly modified and used directly on $\boldsymbol{A}$ instead of (3.3). Particularly, Proposition 3.2 is easy to modify for singular values $\left(\bar{\sigma}_{1}(\boldsymbol{A}) \leq \sigma_{1}(|\boldsymbol{A}|)\right)$, and the interlacing property can be applied directly to $\boldsymbol{A}$; cf. $[13,18,19]$. 
3.6. Case of study. The aim of the following examples is to show that no presented method is better than the others. In different situations, different variants are the best.

Example 3.6. Consider the example given by Qiu, Chen, and Elishakoff [26] (see also [34]):

$$
\boldsymbol{A}^{S}=\left(\begin{array}{cccc}
{[2975,3025]} & {[-2015,-1985]} & 0 & 0 \\
{[-2015,-1985]} & {[4965,5035]} & {[-3020,-2980]} & 0 \\
0 & {[-3020,-2980]} & {[6955,7045]} & {[-4025,-3975]} \\
0 & 0 & {[-4025,-3975]} & {[8945,9055]}
\end{array}\right) .
$$

Proposition 3.2 yields the upper bound $\lambda_{1}^{u}\left(\boldsymbol{A}^{S}\right)=12720.2273$, which is-by chancethe optimal value. The other outer approximations of the eigenvalues sets $\boldsymbol{\lambda}_{i}\left(\boldsymbol{A}^{S}\right)$, $i=1, \ldots, n$, are listed below. The corresponding items are as follows:

(R) bounds computed by Rohn's theorem (Theorem 3.1)

(D1) bounds computed by Algorithm 1 with index selection rule (3.1)

(D2) bounds computed by Algorithm 1 with index selection rule (3.2)

(I1) bounds computed by Algorithm 2 with index selection rule (3.1)

(I2) bounds computed by Algorithm 2 with index selection rule (3.2)

(DD1) bounds computed by diagonal maximization by using Algorithm 1 and index selection rule (3.1)

(DI1) bounds computed by diagonal maximization by using Algorithm 2 and index selection rule (3.1)

(B) bounds obtained by using Theorem 3.1 and Algorithms 1 and 2, and then choosing the best ones; the index selection rule is (3.1)

(O) optimal bounds; they are known provided that an inner and outer approximation (calculated or known from references) coincide; some of them are determined according to Hertz $[15,16]$

TABLE 3.1

Results for Example 3.6.

\begin{tabular}{c|cccc} 
& {$\left[\lambda_{1}^{l}\left(\boldsymbol{A}^{S}\right), \lambda_{1}^{u}\left(\boldsymbol{A}^{S}\right)\right]$} & {$\left[\lambda_{2}^{l}\left(\boldsymbol{A}^{S}\right), \lambda_{2}^{u}\left(\boldsymbol{A}^{S}\right)\right]$} & {$\left[\lambda_{3}^{l}\left(\boldsymbol{A}^{S}\right), \lambda_{3}^{u}\left(\boldsymbol{A}^{S}\right)\right]$} & {$\left[\lambda_{4}^{l}\left(\boldsymbol{A}^{S}\right), \lambda_{4}^{u}\left(\boldsymbol{A}^{S}\right)\right]$} \\
\hline (R) & {$[12560.6296,12720.4331]$} & {$[6984.5571,7144.3606]$} & {$[3309.9466,3469.7501]$} & {$[825.2597,985.0632]$} \\
(D1) & {$[8945.0000,12720.2273]$} & {$[4945.00000,9055.0000]$} & {$[2924.5049,6281.7216]$} & {$[825.2597,3025.0000]$} \\
(D2) & {$[8945.0000,12720.2273]$} & {$[2945.0000,9453.4449]$} & {$[1708.9320,6281.7216]$} & {$[825.2597,3025.0000]$} \\
(I1) & {$[12560.6296,12720.4331]$} & {$[6984.5571,7144.3606]$} & {$[3309.9466,3469.7501]$} & {$[825.2597,985.0632]$} \\
(I2) & {$[12560.6296,12720.4331]$} & {$[6984.5571,7144.3606]$} & {$[3309.9466,3469.7501]$} & {$[825.2597,985.0632]$} \\
(DD1) & {$[8945.0000,12720.2273]$} & {$[4965.0000,9055.0000]$} & {$[2950.0000,6281.7216]$} & {$[837.0637,3025.0000]$} \\
(DI1) & {$[12557.7243,12723.3526]$} & {$[6990.7616,7138.1800]$} & {$[3320.2863,3459.4322]$} & {$[837.0637,973.1993]$} \\
(B) & {$[12560.6296,12720.2273]$} & {$[6990.7616,7138.1800]$} & {$[3320.2863,3459.4322]$} & {$[837.0637,973.1993]$} \\
(O) & {$[12560.8377,12720.2273]$} & {$[7002.2828,7126.8283]$} & {$[3337.0785,3443.3127]$} & {$[842.9251,967.1082]$}
\end{tabular}

Table 3.1 shows that the direct interlacing methods (D1), (D2), and (DD1) are not effective; gaps between the eigenvalue sets $\boldsymbol{\lambda}_{i}\left(\boldsymbol{A}^{S}\right), i=1, \ldots, n$, are too wide. The indirect interlacing methods (I1) and (I2) yield the same intervals as the Rohn method $(\mathrm{R})$. The indirect interlacing method using diagonal maximization is several times better (e.g., for $\lambda_{4}^{l}\left(\boldsymbol{A}^{S}\right), \lambda_{4}^{u}\left(\boldsymbol{A}^{S}\right)$ ) and several times worse (e.g., for $\lambda_{1}^{l}\left(\boldsymbol{A}^{S}\right)$, $\left.\lambda_{1}^{u}\left(\boldsymbol{A}^{S}\right)\right)$ than (R). The combination (B) of all the methods produces good outer approximation of the eigenvalue set, particularly for that of $\boldsymbol{\lambda}_{1}\left(\boldsymbol{A}^{S}\right)$. 
For this example, Qiu, Chen, and Elishakoff [26] obtained the approximate values

$$
\begin{aligned}
& \underline{\lambda}_{1}\left(\boldsymbol{A}^{S}\right) \approx 12588.29, \bar{\lambda}_{1}\left(\boldsymbol{A}^{S}\right) \approx 12692.77, \underline{\lambda}_{2}\left(\boldsymbol{A}^{S}\right) \approx 7000.195, \bar{\lambda}_{2}\left(\boldsymbol{A}^{S}\right) \approx 7128.723, \\
& \underline{\lambda}_{3}\left(\boldsymbol{A}^{S}\right) \approx 3331.162, \bar{\lambda}_{3}\left(\boldsymbol{A}^{S}\right) \approx 3448.535, \underline{\lambda}_{4}\left(\boldsymbol{A}^{S}\right) \approx 826.7372, \bar{\lambda}_{4}\left(\boldsymbol{A}^{S}\right) \approx 983.5858 .
\end{aligned}
$$

However, these values form neither inner nor outer approximations of the eigenvalue set. The method of Leng and He [21] based on matrix perturbation theory results in the following bounds:

$$
\begin{aligned}
& \lambda_{1}^{l}\left(\boldsymbol{A}^{S}\right)=12550.53, \quad \lambda_{1}^{u}\left(\boldsymbol{A}^{S}\right)=12730.53, \quad \lambda_{2}^{l}\left(\boldsymbol{A}^{S}\right)=6974.459, \quad \lambda_{2}^{u}\left(\boldsymbol{A}^{S}\right)=7154.459, \\
& \lambda_{3}^{l}\left(\boldsymbol{A}^{S}\right)=3299.848, \quad \lambda_{3}^{u}\left(\boldsymbol{A}^{S}\right)=3479.848, \quad \lambda_{4}^{l}\left(\boldsymbol{A}^{S}\right)=815.1615, \quad \lambda_{4}^{u}\left(\boldsymbol{A}^{S}\right)=995.1615 .
\end{aligned}
$$

In comparison to (B), they are not so sharp. The evolution strategy method proposed by Yuan, He, and Leng [34] returns an inner approximation of the eigenvalue set, which is equal to the optimal result (see $(\mathrm{O})$ in Table 3.1) in this example.

Example 3.7. Consider the symmetric interval matrix

$$
\boldsymbol{A}^{S}=\left(\begin{array}{ccc}
{[0,2]} & {[-7,3]} & {[-2,2]} \\
{[-7,3]} & {[4,8]} & {[-3,5]} \\
{[-2,2]} & {[-3,5]} & {[1,5]}
\end{array}\right)^{S}
$$

Following the notation used in Example 3.6 we display in Table 3.2 results obtained by the presented methods.

TABLE 3.2

Results for Example 3.7.

\begin{tabular}{c|ccc} 
& {$\left[\lambda_{1}^{l}\left(\boldsymbol{A}^{S}\right), \lambda_{1}^{u}\left(\boldsymbol{A}^{S}\right)\right]$} & {$\left[\lambda_{2}^{l}\left(\boldsymbol{A}^{S}\right), \lambda_{2}^{u}\left(\boldsymbol{A}^{S}\right)\right]$} & {$\left[\lambda_{3}^{l}\left(\boldsymbol{A}^{S}\right), \lambda_{3}^{u}\left(\boldsymbol{A}^{S}\right)\right]$} \\
\hline (R) & {$[-2.2298,16.0881]$} & {$[-6.3445,11.9734]$} & {$[-8.9026,9.4154]$} \\
(D1) & {$[4.0000,15.3275]$} & {$[-2.5616,6.0000]$} & {$[-8.9026,2.0000]$} \\
(D2) & {$[4.0000,15.3275]$} & {$[-2.5616,6.0000]$} & {$[-8.9026,2.0000]$} \\
(I1) & {$[-0.7436,16.0881]$} & {$[-3.3052,10.4907]$} & {$[-8.9026,6.3760]$} \\
(I2) & {$[-0.7436,16.0881]$} & {$[-3.3052,10.4907]$} & {$[-8.9026,6.3760]$} \\
(DD1) & {$[4.0000,15.3275]$} & {$[-2.0000,6.0000]$} & {$[-8.3759,2.0000]$} \\
(DI1) & {$[-0.9115,16.3089]$} & {$[-2.9115,10.8445]$} & {$[-8.3759,6.7850]$} \\
(B) & {$[4.0000,15.3275]$} & {$[-2.0000,6.0000]$} & {$[-8.3759,2.0000]$} \\
(O) & {$[6.3209,15.3275]$} & {$[?, ?]$} & {$[-7.8184,0.7522]$}
\end{tabular}

This example illustrates the case when direct interlacing methods (D1)-(D2) yield better results than the indirect ones (I1)-(I2). The same is true for the diagonal maximization variants (DD1) and (DI1). Rohn's method (R) is not very effective here. Optimal bounds are known only for $\lambda_{1}^{u}\left(\boldsymbol{A}^{S}\right)$ and $\lambda_{3}^{l}\left(\boldsymbol{A}^{S}\right)$. of

Example 3.8. Herein, we consider an example by Deif [9] on singular value sets

$$
\boldsymbol{A}=\left(\begin{array}{ll}
{[2,3]} & {[1,1]} \\
{[0,2]} & {[0,1]} \\
{[0,1]} & {[2,3]}
\end{array}\right)
$$

Deif's method yields the following estimation of the singular value sets:

$$
\boldsymbol{\sigma}_{1}(\boldsymbol{A}) \approx[2.5616,4.5431], \quad \boldsymbol{\sigma}_{2}(\boldsymbol{A}) \approx[1.3134,2.8541] .
$$


Ahn and Chen [1] confirmed that $\bar{\sigma}_{1}(\boldsymbol{A})=4.5431$, but the real value of $\underline{\sigma}_{2}(\boldsymbol{A})$ must be smaller. Namely, it is less than or equal to one since $\sigma_{2}(A)=1$ for $A^{\bar{T}}=\left(\begin{array}{lll}2 & 0 & 1 \\ 1 & 0 & 2\end{array}\right)$. Our approach using a combination of all presented methods results in an outer approximation of

$$
\boldsymbol{\sigma}_{1}(\boldsymbol{A}) \subseteq[2.0489,4.5431], \quad \boldsymbol{\sigma}_{2}(\boldsymbol{A}) \subseteq[0.4239,3.1817] .
$$

4. Conclusion and future work. In this paper we considered outer approximations of the eigenvalue sets of general interval matrices and symmetric interval matrices. For both cases, we presented several improvements. Computing sharp outer approximations of the eigenvalue set of a general interval matrix is a difficult problem. The proposed methods provide quite satisfactory results, as indicated by Examples 2.8-2.9. Examples 3.6-3.8 demonstrate that we are able to bound quite sharply the eigenvalues of symmetric interval matrices and the singular values of interval matrices. Our bounds are quite close to the optimal ones. Nevertheless, as suggested by one of the referees, it is worth exploring the possibility of using a more numerically stable decomposition than the Jordan canonical form in Proposition 2.6.

Currently, there is no algorithm that computes the best bounds in all the cases. Since the computational cost of the presented algorithms is rather low, it is advisable to use all of them in practice and select the best one depending on the particular instance.

Acknowledgments. The authors thank Andreas Frommer and the anonymous referees for their valuable comments.

\section{REFERENCES}

[1] H.-S. Ahn And Y. Q. Chen, Exact maximum singular value calculation of an interval matrix, IEEE Trans. Automat. Control, 52 (2007), pp. 510-514.

[2] G. Alefeld and J. Herzberger, Introduction to Interval Computations, Academic Press, New York, 1983.

[3] G. Alefeld And G. Mayer, Interval analysis: Theory and applications, J. Comput. Appl. Math., 121 (2000), pp. 421-464.

[4] O. Beaumont, An Algorithm for Symmetric Interval Eigenvalue Problem, Technical report IRISA-PI-00-1314, Institut de recherche en informatique et systèmes aléatoires, Rennes, France, 2000.

[5] D. Chablat, Ph. Wenger, F. Majou, and J.-P. Merlet, An interval analysis based study for the design and the comparison of three-degrees-of-freedom parallel kinematic machines, Int. J. Robot. Res., 23 (2004), pp. 615-624.

[6] K.-w. E. ChU, Generalization of the Bauer-Fike theorem, Numer. Math., 49 (1986), pp. 685691.

[7] K.-W. E. Chu, Perturbation theory and derivatives of matrix eigensystems, Appl. Math. Lett., 1 (1988), pp. 343-346.

[8] L. Cvetkovic, V. Kostic, and R. S. Varga, A new Geršgorin-type eigenvalue inclusion set, Electron. Trans. Numer. Anal., 18 (2004), pp. 73-80.

[9] A. S. DeIf, Singular values of an interval matrix, Linear Algebra Appl., 151 (1991), pp. 125133.

[10] A. S. DeIf, The interval eigenvalue problem, Z. Angew. Math. Mech., 71 (1991), pp. 61-64.

[11] A. D. Dimarogonas, Interval analysis of vibrating systems, J. Sound Vibration, 183 (1995), pp. 739-749.

[12] F. Gioia And C. N. Lauro, Principal component analysis on interval data, Comput. Statist., 21 (2006), pp. 343-363.

[13] G. H. Golub and C. F. Van Loan, Matrix Computations, 3rd ed., Johns Hopkins University Press, Baltimore, MD, 1996.

[14] E. Hansen and G. W. Walster, Global Optimization Using Interval Analysis, 2nd ed., Marcel Dekker, New York, 2004. 
[15] D. HerTz, The extreme eigenvalues and stability of real symmetric interval matrices, IEEE Trans. Automat. Control, 37 (1992), pp. 532-535.

[16] D. Hertz, Interval analysis: Eigenvalue bounds of interval matrices, in Encyclopedia of Optimization, C. A. Floudas and P. M. Pardalos, eds., Springer, New York, 2009, pp. 1689-1696.

[17] M. Hladík, D. Daney, and E. Tsigaridas, An Algorithm for the Real Interval Eigenvalue Problem, Research report RR-6680, INRIA, Sophia-Antipolis, France, 2008, http://hal. inria.fr/inria-00329714/en/, submitted to J. Comput. Appl. Math.

[18] R. A. Horn and C. R. Johnson, Matrix Analysis, Cambridge University Press, Cambridge, UK, 1985.

[19] R. A. Horn and C. R. Johnson, Topics in Matrix Analysis, Cambridge University Press, Cambridge, UK, 1994.

[20] L. V. Kolev, Outer interval solution of the eigenvalue problem under general form parametric dependencies, Reliab. Comput., 12 (2006), pp. 121-140.

[21] H. Leng AND Z. He, Computing eigenvalue bounds of structures with uncertain-but-nonrandom parameters by a method based on perturbation theory, Comm. Numer. Methods Engrg., 23 (2007), pp. 973-982.

[22] H.-B. Li, T.-Z. Huang, And H. Li, Inclusion sets for singular values, Linear Algebra Appl., 428 (2008), pp. 2220-2235.

[23] C. D. Meyer, Matrix Analysis and Applied Linear Algebra, SIAM, Philadelphia, 2000.

[24] A. Neumaier, Interval Methods for Systems of Equations, Cambridge University Press, Cambridge, UK, 1990.

[25] S. PoljaK and J. Rohn, Checking robust nonsingularity is NP-hard, Math. Control Signals Systems, 6 (1993), pp. 1-9.

[26] Z. Qiu, S. Chen, And I. Elishakoff, Bounds of eigenvalues for structures with an interval description of uncertain-but-non-random parameters, Chaos Solitons Fractals, 7 (1996), pp. 425-434.

[27] Z. QIU, P. C. Müller, And A. Frommer, An approximate method for the standard interval eigenvalue problem of real non-symmetric interval matrices, Comm. Numer. Methods Engrg., 17 (2001), pp. 239-251.

[28] Z. QIU AND X. WANG, Solution theorems for the standard eigenvalue problem of structures with uncertain-but-bounded parameters, J. Sound Vibration, 282 (2005), pp. 381-399.

[29] J. RoHn, Interval matrices: Singularity and real eigenvalues, SIAM J. Matrix Anal. Appl., 14 (1993), pp. 82-91.

[30] J. Rohn, Bounds on eigenvalues of interval matrices, ZAMM Z. Angew. Math. Mech., 78 (1998), pp. S1049-S1050.

[31] J. Rohn, A Handbook of Results on Interval Linear Problems, http://www.cs.cas.cz/rohn/ handbook (2005).

[32] J. Rohn And A. DeIF, On the range of eigenvalues of an interval matrix, Computing, 47 (1992), pp. 373-377.

[33] J. H. Wilkinson, The Algebraic Eigenvalue Problem, Clarendon Press, Oxford University Press, New York, 1988.

[34] Q. Yuan, Z. He, And H. LenG, An evolution strategy method for computing eigenvalue bounds of interval matrices, Appl. Math. Comput., 196 (2008), pp. 257-265.

Copyright (c) by SIAM. Unauthorized reproduction of this article is prohibited. 